\title{
Generation of Induced Pluripotent Stem Cells and Neural Stem/Progenitor Cells from Newborns with Spina Bifida Aperta
}

\author{
Yohei Bamba ${ }^{1}$, Masahiro Nonaka ${ }^{2}$, Natsu Sasaki $^{3}$, Tomoko Shofuda ${ }^{4}$, Daisuke Kanematsu ${ }^{5}$, Hiroshi Suemizu ${ }^{6}$, \\ Yuichiro Higuchi ${ }^{6}$, Ritsuko K. Pooh ${ }^{7}$, Yonehiro Kanemura ${ }^{5,8}$, Hideyuki Okano ${ }^{1}$, Mami Yamasaki ${ }^{9}$ \\ ${ }^{1}$ Department of Physiology, Keio University School of Medicine, Tokyo, Japan \\ ${ }^{2}$ Department of Pediatric Neurosurgery, Kansai Medical University, Hirakata, Japan \\ ${ }^{3}$ Department of Pediatric Neurosurgery, National Medical Center for Children and Mothers Research Institute, Tokyo, Japan \\ ${ }^{4}$ Division of Stem Cell Research, Institute for Clinical Research, Osaka National Hospital, National Hospital Organization, Osaka, Japan \\ ${ }^{5}$ Division of Regenerative Medicine, Institute for Clinical Research, Osaka National Hospital, National Hospital Organization, Osaka, Japan \\ ${ }^{6}$ Laboratory Animal Research Department, Biomedical Research Laboratory, Central Institute for Experimental Animals, Kawasaki, Japan \\ ${ }^{7}$ CRIFM Clinical Research Institute of Fetal Medicine Pooh Maternity Clinic, Osaka, Japan \\ ${ }^{8}$ Department of Neurosurgery, Osaka National Hospital, National Hospital Organization, Osaka, Japan \\ ${ }^{9}$ Department of Pediatric Neurosurgery, Takatsuki General Hospital, Takatsuki, Japan
}

Study Design: We established induced pluripotent stem cells (iPSCs) and neural stem/progenitor cells (NSPCs) from three newborns with spina bifida aperta (SBa) using clinically practical methods.

Purpose: We aimed to develop stem cell lines derived from newborns with SBa for future therapeutic use.

Overview of Literature: SBa is a common congenital spinal cord abnormality that causes defects in neurological and urological functions. Stem cell transplantation therapies are predicted to provide beneficial effects for patients with SBa. However, the availability of appropriate cell sources is inadequate for clinical use because of their limited accessibility and expandability, as well as ethical issues.

Methods: Fibroblast cultures were established from small fragments of skin obtained from newborns with SBa during SBa repair surgery. The cultured cells were transfected with episomal plasmid vectors encoding reprogramming factors necessary for generating iPSCs. These cells were then differentiated into NSPCs by chemical compound treatment, and NSPCs were expanded using neurosphere technology.

Results: We successfully generated iPSC lines from the neonatal dermal fibroblasts of three newborns with SBa. We confirmed that these lines exhibited the characteristics of human pluripotent stem cells. We successfully generated NSPCs from all SBa newbornderived iPSCs with a combination of neural induction and neurosphere technology.

Conclusions: We successfully generated iPSCs and iPSC-NSPCs from surgical samples obtained from newborns with SBa with the goal of future clinical use in patients with SBa.

Keywords: Myelomeningocele; Spina bifida; Induced pluripotent stem cells; Neural stem/progenitor cells; Regenerative medicine

Received Mar 28, 2017; Revised Apr 18, 2017; Accepted Apr 22, 2017

Corresponding author: Yohei Bamba

Department of Physiology, Keio University School of Medicine, 35 Shinanomachi, Shinjuku-ku, Tokyo 160-8582, Japan

Tel: +81-072-252-3561, Fax: +81-072-252-3346, E-mail: ybamba@osakah.johas.go.jp 


\section{Introduction}

Spina bifida aperta (SBa; we primarily refer to the most severe form of SBa, myelomeningocele) is one of the most common human congenital abnormalities. $\mathrm{SBa}$ is caused by incomplete neural tube closure, followed by the development of open vertebral arches and skin lesions during early fetal development. Unprotected neural tissues exposed to amniotic fluid are damaged and fail to develop properly, leading to neurological dysfunction below the level of the spinal cord defect.

Because of the issues associated with traditional and available experimental therapies, regenerative therapy is currently under investigation for treatment of patients with SBa. The recent development of induced pluripotent stem cells (iPSCs) [1], which can self-renew, proliferate, and differentiate into various types of cells or tissue and are expandable, has facilitated the advancement of regenerative medicine. Many studies are being conducted using iPSCs for the treatment of spinal cord injuries (SCIs), retinal diseases, and neurodegenerative disorders $[2,3]$. Considering that SBa-related complications occur over a long period, a sufficient amount of therapeutic cells would need to be obtained as soon as possible after birth. We generated and characterized iPSCs and iPSCs-derived neural stem/progenitor cells (NSPCs) from newborns with SBa for future clinical application.

\section{Materials and Methods}

\section{Patients}

We recruited patients for our study in collaboration with the Fetal Brain Malformation Network, which consists of Japanese investigators who specialize in research on congenital central nervous system malformations. We established primary fibroblast cultures and iPSCs from three newborns with SBa (patient 3, 8, 34) after obtaining informed consent from parents. These newborns were diagnosed with lumbosacral myelomeningocele by specialized obstetricians using prenatal ultrasonography. All newborns were females born at 38 (patient 3,34 ) or 37 (patient 8) weeks of gestation and were delivered by planned cesarean section.

This study was conducted in accordance with the principles of the Declaration of Helsinki and was approved by the ethics committees of Osaka National Hospital (Nos.
123 and 146) with appropriate informed consent including participation and publication.

\section{Surgical repair of myelomeningocele}

All SBa repair surgeries were performed with the patient under general anesthesia within the first few hours of birth by a skilled pediatric neurosurgeon. The neural placode was dissected from the surrounding tissue at the transitional border. After careful removal of epidermal tissue from the rim of the open neural placode and spinal cord, the surrounding structures were reconstructed using the five-layer closure method. During repair of the skin defects and closure of the opening, superfluous dermal tissue at the edge of the wound was excised to prepare the suture plane. These fragments of excised skin were used to establish primary fibroblast cultures.

\section{Isolation of SBa patient-derived fibroblasts}

The fragments of surgically removed skin (approximately $3 \times 5-10 \mathrm{~mm}$ ) were separated from the subcutaneous tissue and cut into small pieces (approximately $1-2 \mathrm{~mm}$ in diameter). They were then digested in a solution containing $50 \mu \mathrm{g} / \mathrm{mL}$ type I collagenase (Life Technologies, Carlsbad, CA, USA) and $50 \mu \mathrm{g} / \mathrm{mL}$ dispase (Life Technologies) at $37^{\circ} \mathrm{C}$ for 30 minutes. The extracted cells were collected by centrifugation and seeded in a cell culture flask with Dulbecco's modified Eagle's medium (DMEM)/F12 (SigmaAldrich, St. Louis, MO, USA) containing 15 mM HEPES, $1 \%$ antibiotic-antimycotic (Life Technologies), and 10\% fetal bovine serum (Life Technologies), as described previously [4].

\section{Establishment of patient-derived fibroblast cultures and iPSCs}

The skin-derived fibroblasts $\left(1.0 \times 10^{6}\right.$ cells $)$ were nucleofected with three plasmids, pCXLE-hSK, pCXLEhOCT3/4-shp53-F, and pCXLE-hUL [5]. These plasmids encode the reprogramming factors SOX2 and KLF4, OCT3/4, and short hairpin RNA against p53, LIN28, and L-MYC, respectively. All plasmids were kindly gifted by Prof. Shinya Yamanaka of Kyoto University to Y.K. and H.O. Nucleofection was performed using a human neonatal fibroblast nucleofection kit (Lonza, Basel, Switzerland) and the Amaxa Nucleofector I device (Lonza), set 
to program U-020, in accordance with the manufacturer's protocol. The transfected cells were seeded in a $6-\mathrm{cm}$ cell culture dish. After 7 days, the cells were harvested and seeded on a layer of mouse embryonic fibroblast feeder cells (Kitayama Labes, Kyoto, Japan) in $5 \times 10^{4}$ to $5 \times 10^{5}$ cells $/ 10-\mathrm{cm}$ dish. At approximately 28 days after nucleofection, human embryonic stem cell (ESC)-like colonies were picked and cultured in ESC cell medium as previously described [6]. At passages 11-14, karyotyping analysis was performed by conventional Giemsa staining and G-banding [6]. Teratoma formation in NOD/Shiscid/IL-2Rg null (NOG) mice was performed at the Central Institute for Experimental Animals (CIEA; Kawasaki, Japan) using the guidelines of the Animal Care Committee at CIEA [7]. The procedure was also approved by the Animal Care Committee at CIEA. Control human iPSCs (hiPSCs; clone 201B7) were obtained from the RIKEN cell bank (RIKEN, Tsukuba, Japan).

\section{Neural induction and neurosphere expansion}

A highly efficient neural induction method based on dual SMAD-inhibition was used as described previously [8] with slight modifications. Briefly, hiPSCs cultured on growth-factor-reduced Matrigel (BD Biosciences, San Jose, CA, USA)-coated dishes were suspended in lowattachment dishes (Sumitomo Bakelite, Tokyo, Japan) to generate embryoid bodies (EBs). For neural differentiation, EBs were cultured in DMEM/F12 containing 5\% B27 supplement (Life Technologies), 5\% N2 supplement (Life Technologies), $20 \mathrm{ng} / \mathrm{mL}$ recombinant human basic fibroblast growth factor (bFGF; Wako, Osaka, Japan), $10 \mu \mathrm{M}$ SB431542 (Sigma-Aldrich), and $1 \mu \mathrm{M}$ dorsomorphin (Wako) under $5 \% \mathrm{O}_{2}$ for 2 weeks. To generate neurospheres, EBs were mechanically disrupted and seeded in DMEM/F12 containing 2\% B27 supplement, $20 \mathrm{ng} /$ mL bFGF (PeproTech, Rocky Hill, NJ, USA), 20 ng/mL epidermal growth factor (EGF; PeproTech), $5 \mu \mathrm{g} / \mathrm{mL}$ heparin (Sigma-Aldrich), and $10 \mathrm{ng} / \mathrm{mL}$ human leukemia inhibitory factor (LIF; Millipore, Billerica, MA, USA), as previously described [9]. After 4-8 passages, the nonadherent floating neurospheres that stably proliferated were analyzed as described below. Neurospheres were plated onto a Matrigel-coated plate in neurobasal medium (Life Technologies) containing 2\% B27 supplement (Life Technologies) and $2 \mathrm{mM} \mathrm{L}$-glutamine (Life Technologies) to determine the efficiency of neural conversion.

\section{Immunocytochemistry}

The cells were fixed with $4 \%$ paraformaldehyde for 15 minutes at $4^{\circ} \mathrm{C}$, followed by permeabilization with $0.3 \%$ Triton X/phosphate buffered saline (PBS) for 5 minutes. To block nonspecific binding, the cells were incubated in $0.01 \%$ Triton X/PBS with $10 \%$ goat serum for 60 minutes. The blocked cells were then incubated at $4^{\circ} \mathrm{C}$ with primary antibodies (see below) overnight, washed with PBS three times, and incubated with secondary antibodies (see below) for 30 minutes. Nuclear staining was performed with TO-PRO-3 iodide (1:1,000, Life Technologies) or 4' 6-diamidino-2-phenylindole (1:1,000). Fluorescent images were acquired by confocal laser microscopy with an LSM510 (Carl Zeiss, Oberkochen, Germany) or fluorescence microscopy with an IX81 microscope (Olympus, Tokyo, Japan). The following primary antibodies were used in this study: mouse anti-Oct3/4 (1:200, BD Biosciences), rabbit anti-nanog (1:200, ReproCELL, Kanagawa, Japan), rabbit anti-SSEA3 (1:200, Millipore), rabbit antiSSEA4 (1:200, Millipore), mouse anti-TRA-1-60 (1:200, Millipore), mouse anti-TRA-1-81 (1:200, Millipore), rabbit anti-Nestin (1:500, prepared in-house [10]), mouse anti-beta III tubulin (1:500, Covance, Inc., Princeton, NJ, USA), mouse anti-PAX6 (1:200, Developmental Studies Hybridoma Bank, University of Iowa, Iowa City, IA, USA), and rabbit anti-GFAP (1:80, Sigma-Aldrich). The following secondary antibodies from Invitrogen (Carlsbad, CA, USA) were used: Alexa Fluor 488-labeled goat antimouse IgG (1:1,000), IgG Alexa Fluor 568-labeled goat anti-rabbit IgG $(1: 1,000)$, Alexa Fluor 488-labeled goat anti-rat IgM $(1: 1,000)$, and IgM Alexa Fluor 568-labeled goat anti-mouse IgM $(1: 1,000)$.

\section{Genomic DNA isolation and polymerase chain reac- tion for episomal vector detection}

Genomic DNA was extracted from each iPSC clone using the DNeasy Blood \& Tissue Kit (Qiagen, Venulo, Netherlands). The polymerase chain reaction (PCR) conditions and primer sequences were used as previously described [5].

\section{RNA extraction and quantitative reverse transcrip- tion polymerase chain reaction}

RNA was extracted from undifferentiated iPSCs, neu- 
ral aggregates, and proliferating neurospheres using the RNeasy Mini Kit (Qiagen). cDNAs were synthesized using the PrimeStarRT Reagent Kit (Takara Bio., Shiga, Japan). Quantitative reverse transcription polymerase chain

Table 1. Primer sequences

\begin{tabular}{|c|c|c|}
\hline Primer name & Use & Sequence \\
\hline GAPDH-F & qPCR & CCACTTTGTCAAGCTCATTTCCT \\
\hline GAPDH-R & qPCR & TCTCTTCCTCTTGTGCTCTTGCT \\
\hline NESTIN-F & qPCR & CCAAGACTGCCCTGGAAAC \\
\hline NESTIN-R & qPCR & ССТСССTCTCCAAGGAAACA \\
\hline NANOG-F & qPCR & GCAGAAGGCCTCAGCACCTA \\
\hline NANOG-R & qPCR & GGTTCCCAGTCGGGTTCAC \\
\hline SOX2-F & qPCR & GGGAAATGGGGAGGGGTGCAAAAGAGG \\
\hline SOX2-R & qPCR & TTGCGTGAGTGTGGATGGGATTGGTG \\
\hline DNMT3B-F & qPCR & TGCTGCTCACAGGGCCCGATACTTC \\
\hline DNMT3B-R & qPCR & TCCTTTCGAGCTCAGTGCACCACAAAAC \\
\hline ОСТ3/4-F & qPCR & GACAGGGGGAGGGGAGGAGCTAGG \\
\hline OCT3/4-R & qPCR & СТTCССТCСАAСCAGTTGCCCCAAAC \\
\hline PAX6-F & qPCR & ACCTGGCTAGCGAAAAGCAA \\
\hline PAX6-R & qPCR & CCCGTTCAACATCCTTAGTTTATCA \\
\hline pEP4-SR & PCR & TCGGGGGTGTTAGAGACAAC \\
\hline pEP4-SF & PCR & TTCCACGAGGGTAGTGAACC \\
\hline
\end{tabular}

qPCR, quantitative polymerase chain reaction; PCR, polymerase chain reaction.

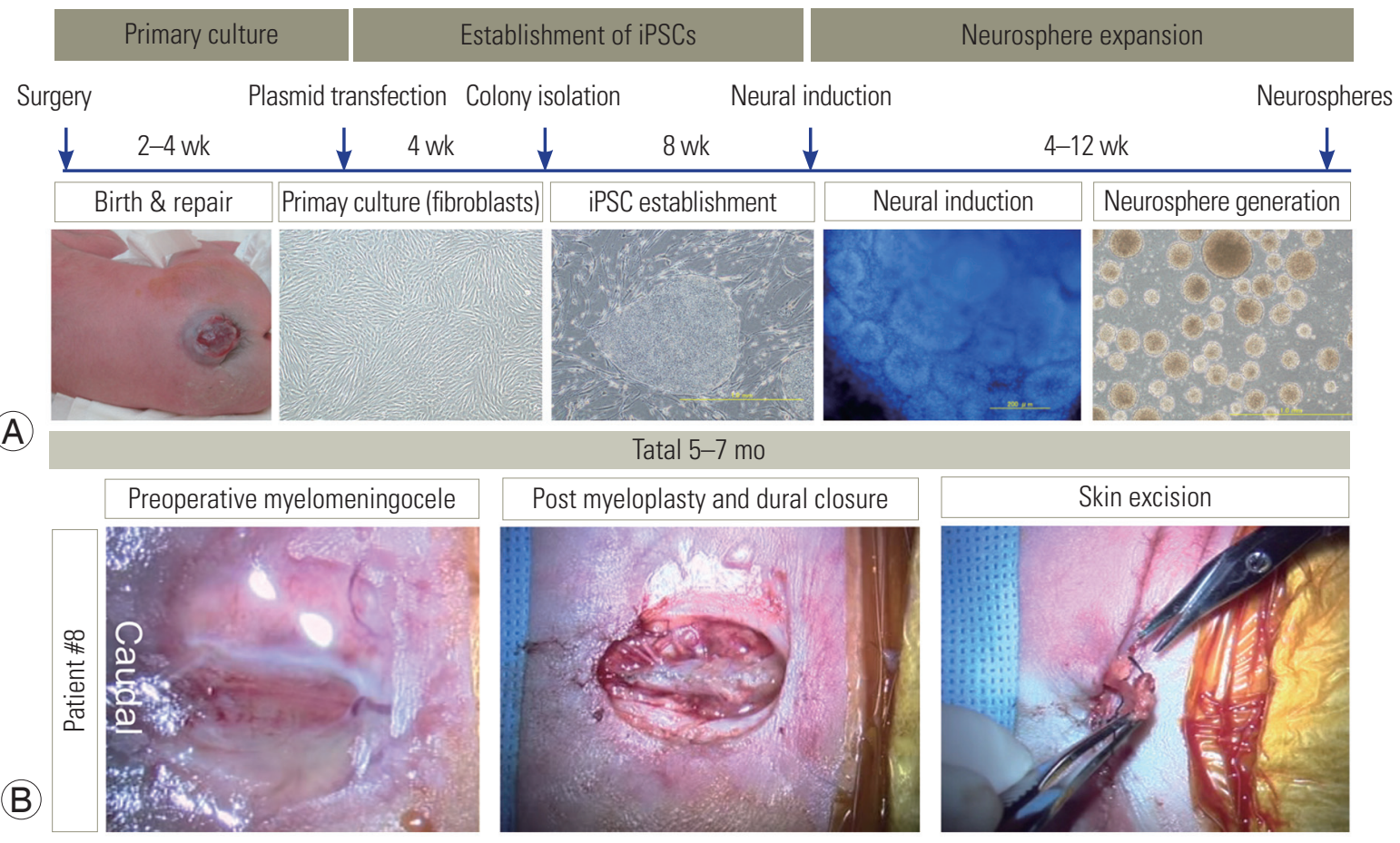

Fig. 1. Generation of induced pluripotent stem cells (iPSC) and iPSC-derived neural stem/progenitor cells from spina bifida aperta patient-derived dermal fibroblasts. (A) Time frame for generating patient-derived neurospheres from surgical specimens. Representative photographs of each stage in the process. (B) Photographs represent the surgical site before incision, the site after myeloplasty and duraplasty, and skin sampling procedure. 
reaction for determining marker gene expression was performed using the Power SYBER Green PCR Master Mix (Life Technologies) and analyzed with the ABI 7300 Real-Time PCR system (Life Technologies). The relative expression of each gene was calculated with the ddCT algorithm using glyceraldehyde-3-phospate dehydrogenase as an internal control, as previously described [11]. The primer set sequences are shown in Table 1.

\section{Results}

\section{Isolation of dermal fibroblasts from the surgically excised skin of newborns with SBa}

SBa repair procedures were performed within the first few hours of birth. In all cases, the skin fragments were collected, and dermal fibroblast cultures were established and expanded without contaminating it. The time frame for the generation of fibroblasts, iPSCs, and neurospheres derived from the skin specimens of newborns with $\mathrm{SBa}$ is shown (Fig. 1A). The entire process took approximately 6 months. Representative photographs of the repair process for patient 8 showed that during skin closure, small skin fragments were excised in preparation for the skin suture. These skin fragments were used to establish primary fibroblast cultures (Fig. 1B).

\section{Generation of SBa patient-derived iPSCs}

Three episomal plasmids encoding cellular reprogramming factors were introduced into the SBa patient-derived fibroblasts by nucleofection. Approximately 3 weeks after transfection, human ESC-like colonies were visible, and 1 week later, the colonies were isolated and expanded. We established three iPSC lines (iPS\#3, \#8, \#34 from patient $3,8,34$, respectively). The established iPSC lines were analyzed by immunohistochemistry and exhibited an ESC-like morphology and expressed ESC markers (Fig.

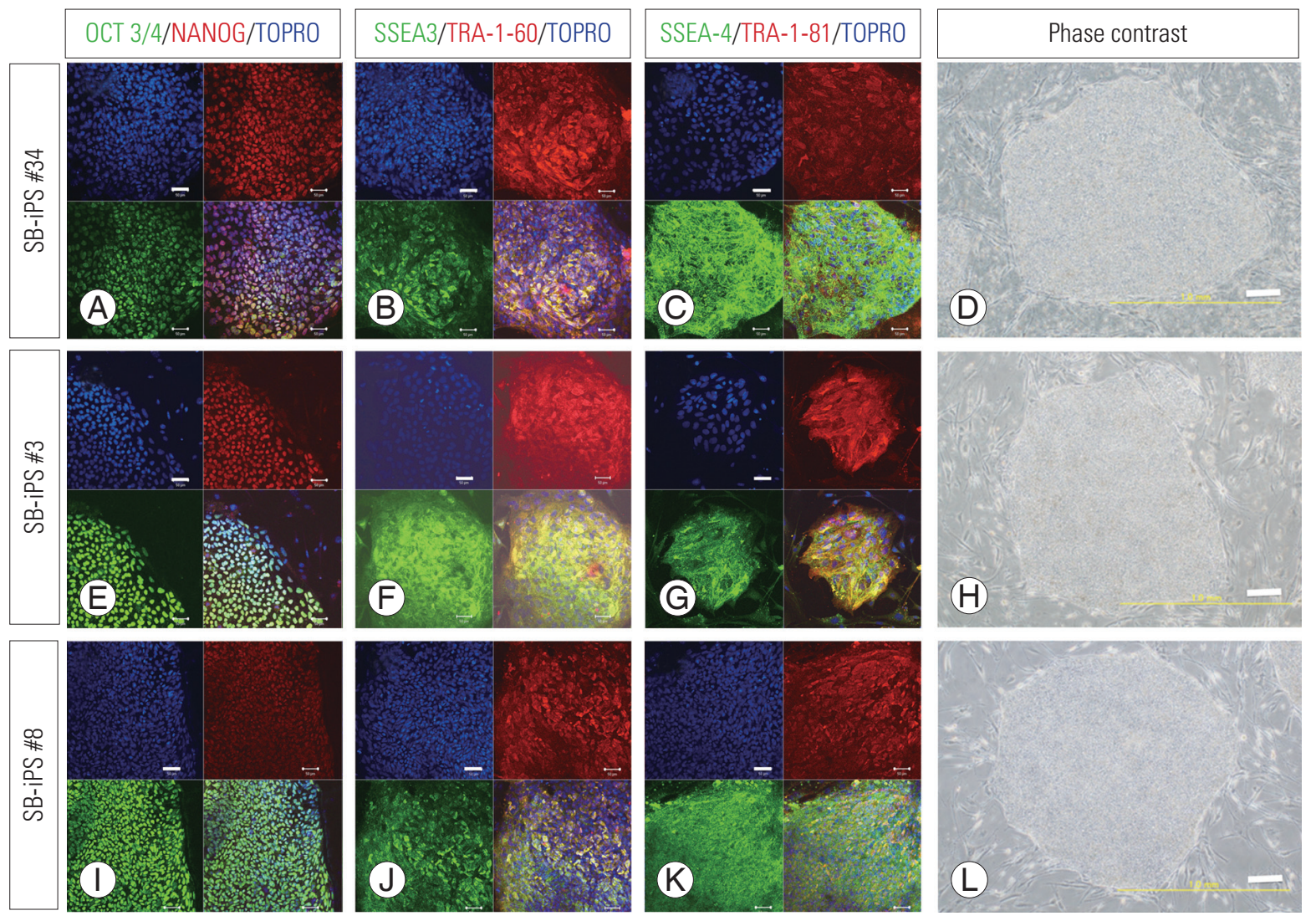

Fig. 2. Immunocytochemistry of spina bifida aperta (SBa) patient-derived induced pluripotent stem cells (iPSCs). Immunostaining of three iPSC lines (iPS \#34, \#3, and \#8) from three different newborns with SBa. (A, E, I) OCT3/4 and NANOG. (B, F, J) SSEA3 and TRA-1-60. (C, G, K) SSEA4 and TRA-1-81. Scale bar=50 $\mu \mathrm{m}$. Lower-right panels in each set of micrographs in (A-K) are merged images. (D, H, L) Phasecontrast microscopic images of the patient-derived iPSC lines. Scale bar=200 $\mu \mathrm{m}$. The morphologies and immunocytochemical features of the three iPSC lines were typical of pluripotent stem cells. 

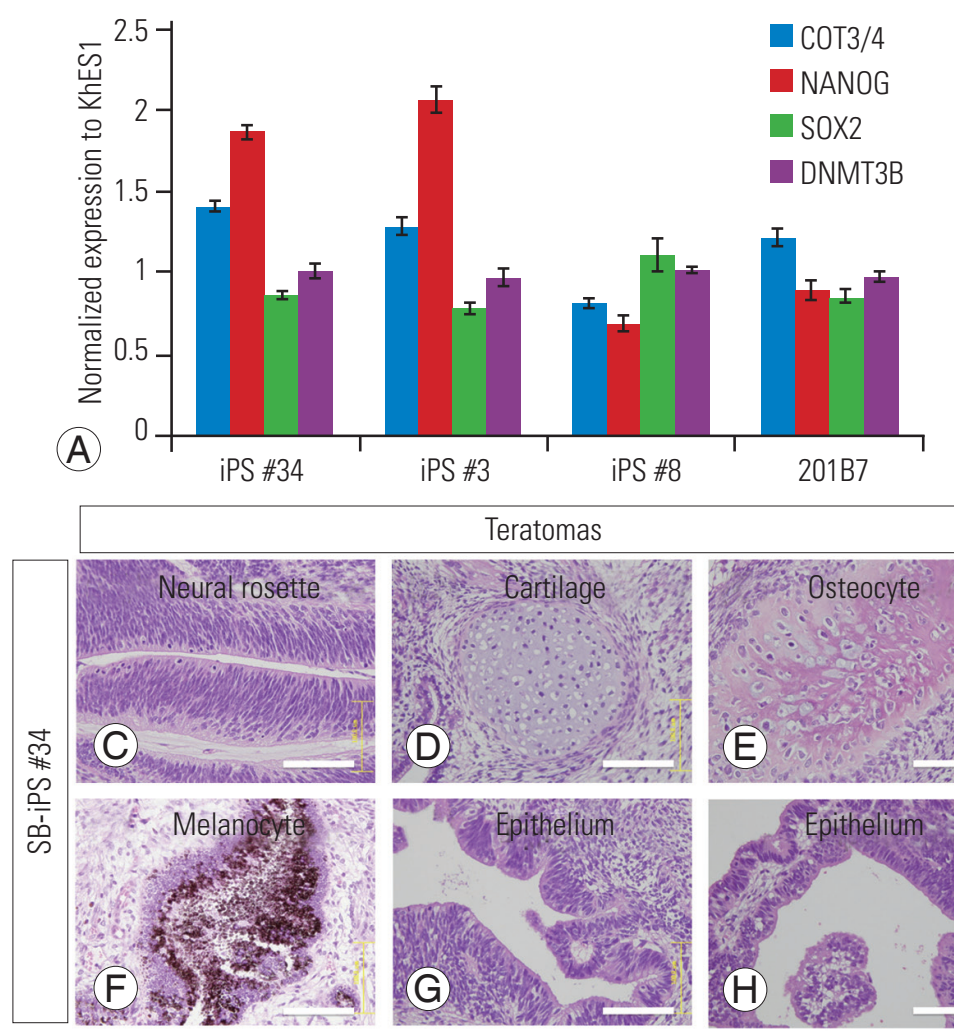

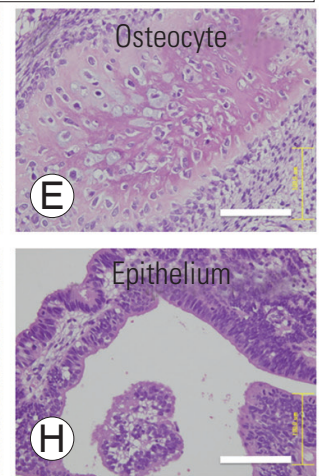

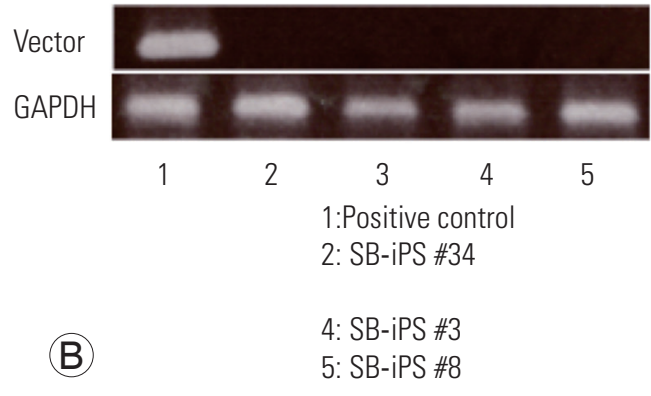
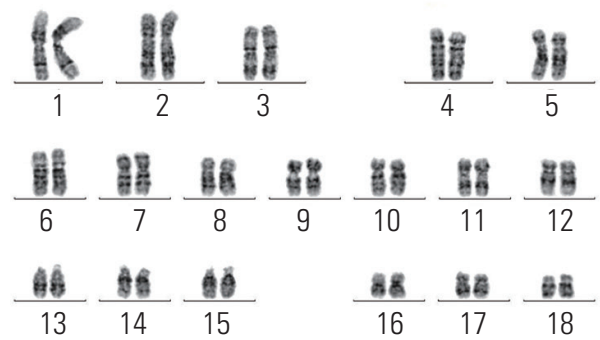

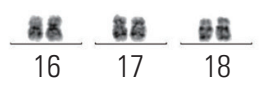

(1) $19 \frac{8}{20}$

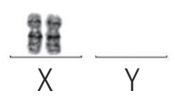

Fig. 3. Characterization of spina bifida aperta (SBa) patient-derived induced pluripotent stem cells (iPSCs). (A) Quantitative polymerase chain reaction (PCR) analysis of pluripotency-associated gene expression in the iPSC lines. The expression values were normalized to those of the control embryonic stem cell line, KhES1 (mean \pm SD). (B) Conventional PCR for detecting the plasmid vector. No signals were detected in any iPSC lines with the primer set EP4 (lane No. 1, positive control; No. 2, iPS \#34; No. 3, not used in this study; No. 4, iPS \#3; No. 5, iPS\#8). (C-H) Hematoxylin and eosin staining of subcutaneously formed teratomas in NOG mice following the transfer of iPS \#34 cells. The cell/tissue composition of the teratomas included neural rosettes (C), cartilage (D), osteocytes (E), melanocytes (F), and various types of epithelium (G, H). Neural rosettes in the teratomas exhibited aligned and well-organized structures (C). Scale bars=100 $\mu \mathrm{m}$. (I) Karyotype of iPS \#34 cells shows that the SBa patient-derived iPSCs exhibit normal (46XX) karyotypes.

2). Real-time PCR analysis showed that the iPSC clones expressed pluripotent stem cell genes at levels similar to those of the control iPSC line, 201B7, and the control human ESC line, KhES1 (Fig. 3A). The KhES1 expression data shown in Fig. 3A were previously reported by our group [12]. We also showed that the episomal plasmid vector used for reprogramming was undetectable by PCR (Fig. 3B). To confirm their pluripotency, the iPS\#34 cells were subcutaneously transplanted into NOG mice. The transplanted iPSCs formed well-differentiated teratomas consisting of three germ layers (Fig. 3C-H). And iPSCs exhibited normal (46XX) karyotypes (Fig. 3I). Thus, we concluded that the SBa patient-derived iPSCs exhibited characteristics of ESCs. Unexpectedly, the SBa-derived teratomas contained well-aligned and closed neural tubelike structures (Fig. 3C), even though their donors exhibited incompletely closed neural tubes.

\section{Neural induction and neurosphere formation from SBa patient-derived iPSCs}

To obtain the amount of cells required for future clinical application, we developed the two-step high efficient neurosphere production system, which consists of neural induction and neurosphere expansion. The exposure of SB patient-derived iPSCs to dual SMAD inhibition for 2 weeks [8] promoted the induction of neural progenitor cells. To produce large numbers of patient-derived NSPCs, which would be required for future clinical applications, we inserted an NSPC propagation step after neural induction, in which NSPCs were cultured in a neurosphere selfrenewal medium containing bFGF, EGF, and LIF (Fig. $4 \mathrm{~A})$. For each of the cultured NSPC lines, we successfully obtained expandable NSPCs as neurospheres. Quantitative PCR of the neurospheres showed that they expressed the neural stem cell (NSC) markers NESTIN and SOX2 
Formation of $\mathrm{EB}$ $\downarrow \quad$ Neural induction

14 days
(A) (with SMAD inhibitors
Dissociation

$\downarrow$ (with bFGF, EGF, LIF)

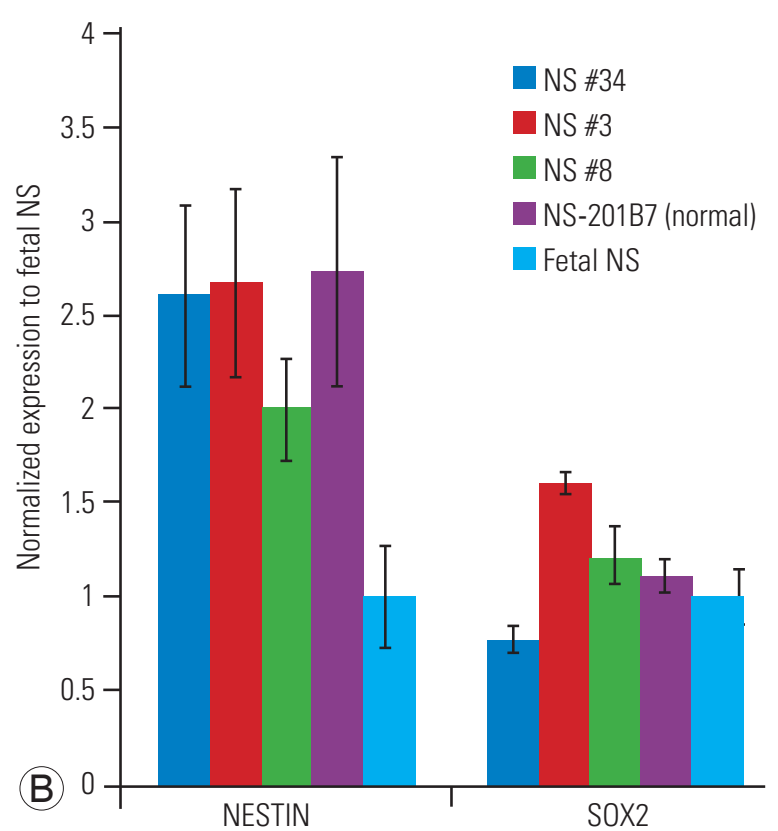

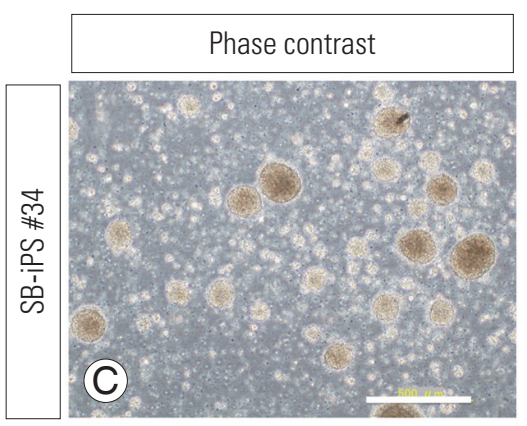

BIII tubulin/GFAP/TOPRO
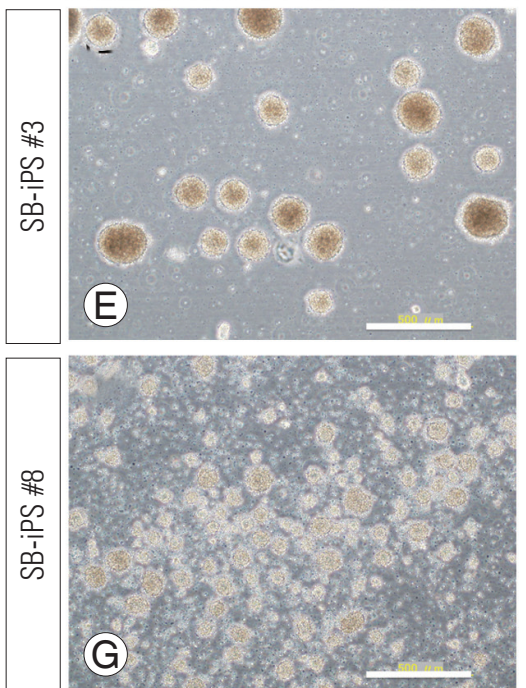
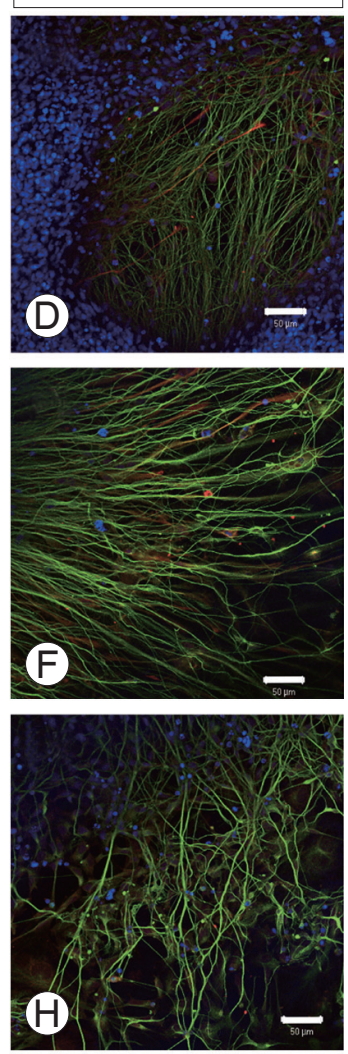

Fig. 4. Generation of spina bifida aperta patient-derived neurospheres. (A) Time frame for neural induction and generation and propagation of patient-derived neurospheres. Dual SMAD inhibition involved the use of SB431542 and dorsomorphin. (B) Quantitative polymerase chain reaction analysis of neural stem (NS) cell marker expression in patient-derived neurospheres. Marker expression in each neurosphere culture was normalized to that of neurospheres derived from the human forebrain-derived NS cell line, oh-NSC-3-fb (means \pm SD). NS \#34, \#3, \#8, 201B7 were neurospheres derived from iPS \#34, \#3, \#8, 201B7 (control induced pluripotent stem cells line), respectively. (C-H) Phase-contrast images of each neurosphere culture and immunostaining for neuronal (B3 tubulin) and glial marker (GFAP) after differentiation of neurospheres. Scale bars=200 $\mu \mathrm{m}$ (C, E, G), 50 $\mu \mathrm{m}$ (D, F, H).

at levels similar to those expressed in the human NSC line oh-NSC-3-fb [9] (Fig. 4B). These neurospheres (Fig. $4 \mathrm{C}, \mathrm{E}, \mathrm{G}$ ) could be passaged and could differentiate into neuronal and glial lineages (Fig. 4D, F, H). Therefore, SBaderived neurospheres could be generated and propagated using our method.

\section{Discussion}

The transplantation of iPSC-derived NSPCs in animal models of SCI is well described and results in functional recovery. Transplanted neural progenitors produce neurotropic factors, myelinate host neurons, and differentiate into neurons that form functional synapses with host neurons [13-17]. Patients with SBa suffer from spinal cord dysfunction, which may be partly due to amniotic fluid exposure following defective neural tube development
[18]. Because the plasticity of the central nervous system is greatest during childhood, we reasoned that human stem cell-based transplantation for children with SBa may be a promising therapeutic approach. In support of this possibility, it has been reported that the transplantation of undifferentiated human ESCs [19] or iPSC-derived neural crest stem cells [20] into the injured spinal cord in an animal model of myelomeningocele results in functional improvement.

We showed that iPSCs could be generated from the skin of newborns with $\mathrm{SBa}$ and that it was possible to generate the numbers of NSPCs required for spinal cord regeneration. This study suggested that iPSC-based autologous transplantation therapy for patients with $\mathrm{SBa}$ is feasible. However, preclinical transplantation studies will be required to establish the safety and efficacy of this therapy. Autologous iPSC-derived cells are expected to be mini- 
mally immunogenic [21-23]. Thus, the transplantation of these cells should not require any additional immunosuppressive therapy, an advantage for patients with SBa undergoing cell transplantation therapy at an early age.

Other types of somatic stem cells, including NSCs, amniotic fluid stem cells (AFSCs), and bone marrow-derived mesenchymal stem cells (BM-MSCs) have also been considered as possible sources for regenerative therapy in SBa [24-26]. NSCs and mesenchymal stem cells (MSCs) were used in animal model transplantation studies with promising results $[24,26]$. AFSCs can be easily isolated, and their use is associated with fewer ethical issues. In addition, AFSCs have the potential to differentiate into neural cells. If techniques for large-scale propagation of AFSCs are developed, they may become a useful cell source for regenerative approaches in SBa. BM-MSCs may also represent a clinically useful cell source; clinical procedures for their isolation have been established, and they are also capable of neuronal differentiation. Furthermore, a recent report indicates that human BM-MSCs can contribute to bladder regeneration [27]. However, invasive procedures, which are not easy to perform on newborns, are required to collect these cells from iliac or femoral bone. If MSCs could be generated from patient-derived iPSCs, they would represent a more promising cell source for treating patients with SBa. Further studies will be required to determine which cell sources are most useful and practical for treating patients with $\mathrm{SBa}$.

One concern regarding SBa-derived iPSCs is that their therapeutic potential could be limited because of possible genetic alterations associated with SBa. However, sporadic $\mathrm{SBa}$ is a multifactorial disease, the development of which is largely affected by environmental factors. However, we successfully generated NSPCs that expand and differentiate normally in vitro from all SBa newborn-derived iPSCs. Thus, we believe that some defects of SBa newborn-derived iPSCs, even if present, could be overcome by culture conditions, and SBa newborn-derived NSPCs then could be obtained stably from subsequent neurosphere culture after neural induction.

Finally, while our study was aimed at mainly developing a practical method for generating SBa newbornderived iPSCs and NSPCs, we have not demonstrated the therapeutic potential of these iPSC-derived NSPCs until now. Therefore, although we cannot currently discuss its clinical application, the accumulating evidence concerning the efficacy and safety of iPSC-derived cells $[28,29]$ encourages us to advance this basic study toward stem cell transplantation therapy in patients with SBa.

\section{Conclusions}

We successfully established SBa newborn-derived iPSC lines and iPSC-derived NSPCs from skin fragments obtained during SBa postnatal repair surgery. We also developed a practical method to generate NSPCs from SBa newborn-derived iPSCs, which may be useful for advancing cell transplantation therapy for patients with SBa.

\section{Conflict of Interest}

No potential conflict of interest relevant to this article was reported.

\section{Acknowledgments}

We thank all members of the Okano and Kanemura laboratories for useful discussions and technical assistance. This work was supported by Research on Applying Health Technology, Health, and Labor Sciences Research Grants to M.Y. from the Ministry of Health, Labor, and Welfare of Japan and the Program for Intractable Disease Research Utilizing Disease-specific iPS Cells funded by the Japan Science and Technology Agency (JST)/Japan Agency for Medical Research and Development (A-MED) to H.O.

\section{References}

1. Takahashi K, Tanabe K, Ohnuki M, et al. Induction of pluripotent stem cells from adult human fibroblasts by defined factors. Cell 2007;131:861-72.

2. Kamao H, Mandai M, Okamoto S, et al. Characterization of human induced pluripotent stem cell-derived retinal pigment epithelium cell sheets aiming for clinical application. Stem Cell Reports 2014;2:205-18.

3. Doi D, Samata B, Katsukawa M, et al. Isolation of human induced pluripotent stem cell-derived dopaminergic progenitors by cell sorting for successful transplantation. Stem Cell Reports 2014;2:337-50.

4. Kanematsu D, Shofuda T, Yamamoto A, et al. Isolation and cellular properties of mesenchymal cells derived from the decidua of human term placenta. Differentiation 2011;82:77-88.

5. Okita K, Matsumura Y, Sato Y, et al. A more efficient 
method to generate integration-free human iPS cells. Nat Methods 2011;8:409-12.

6. Shofuda T, Kanematsu D, Fukusumi H, et al. Human decidua-derived mesenchymal cells are a promising source for the generation and cell banking of human induced pluripotent stem cells. Cell Med 2013;4:12547.

7. Ito M, Hiramatsu H, Kobayashi K, et al. NOD/ SCID/gamma(c)(null) mouse: an excellent recipient mouse model for engraftment of human cells. Blood 2002;100:3175-82.

8. Wang J, Gallagher D, DeVito LM, et al. Metformin activates an atypical PKC-CBP pathway to promote neurogenesis and enhance spatial memory formation. Cell Stem Cell 2012;11:23-35.

9. Kanemura Y, Mori H, Kobayashi S, et al. Evaluation of in vitro proliferative activity of human fetal neural stem/progenitor cells using indirect measurements of viable cells based on cellular metabolic activity. J Neurosci Res 2002;69:869-79.

10. Nakamura Y, Yamamoto M, Oda E, et al. Expression of tubulin beta II in neural stem/progenitor cells and radial fibers during human fetal brain development. Lab Invest 2003;83:479-89.

11. Shofuda T, Fukusumi H, Kanematsu D, et al. A method for efficiently generating neurospheres from human-induced pluripotent stem cells using microsphere arrays. Neuroreport 2013;24:84-90.

12. Fukusumi H, Shofuda T, Kanematsu D, et al. Feederfree generation and long-term culture of human induced pluripotent stem cells using pericellular matrix of decidua derived mesenchymal cells. PLoS One 2013;8:e55226.

13. Tsuji O, Miura K, Okada Y, et al. Therapeutic potential of appropriately evaluated safe-induced pluripotent stem cells for spinal cord injury. Proc Natl Acad Sci U S A 2010;107:12704-9.

14. Yasuda A, Tsuji O, Shibata S, et al. Significance of remyelination by neural stem/progenitor cells transplanted into the injured spinal cord. Stem Cells 2011;29:1983-94.

15. Nori S, Okada Y, Yasuda A, et al. Grafted humaninduced pluripotent stem-cell-derived neurospheres promote motor functional recovery after spinal cord injury in mice. Proc Natl Acad Sci U S A 2011;108:16825-30.

16. Fujimoto Y, Abematsu M, Falk A, et al. Treatment of a mouse model of spinal cord injury by transplantation of human induced pluripotent stem cell-derived long-term self-renewing neuroepithelial-like stem cells. Stem Cells 2012;30:1163-73.

17. Kobayashi Y, Okada Y, Itakura G, et al. Pre-evaluated safe human iPSC-derived neural stem cells promote functional recovery after spinal cord injury in common marmoset without tumorigenicity. PLoS One 2012;7:e52787.

18. Stiefel D, Meuli M. Scanning electron microscopy of fetal murine myelomeningocele reveals growth and development of the spinal cord in early gestation and neural tissue destruction around birth. J Pediatr Surg 2007;42:1561-5.

19. Lee DH, Park S, Kim EY, et al. Enhancement of reclosure capacity by the intra-amniotic injection of human embryonic stem cells in surgically induced spinal open neural tube defects in chick embryos. Neurosci Lett 2004;364:98-100.

20. Saadai P, Wang A, Nout YS, et al. Human induced pluripotent stem cell-derived neural crest stem cells integrate into the injured spinal cord in the fetal lamb model of myelomeningocele. J Pediatr Surg 2013;48:158-63.

21. Guha P, Morgan JW, Mostoslavsky G, Rodrigues NP, Boyd AS. Lack of immune response to differentiated cells derived from syngeneic induced pluripotent stem cells. Cell Stem Cell 2013;12:407-12.

22. Araki R, Uda M, Hoki Y, et al. Negligible immunogenicity of terminally differentiated cells derived from induced pluripotent or embryonic stem cells. Nature 2013;494:100-4.

23. Morizane A, Doi D, Kikuchi T, et al. Direct comparison of autologous and allogeneic transplantation of iPSC-derived neural cells in the brain of a nonhuman primate. Stem Cell Reports 2013;1:283-92.

24. Fauza DO, Jennings RW, Teng YD, Snyder EY. Neural stem cell delivery to the spinal cord in an ovine model of fetal surgery for spina bifida. Surgery 2008;144:367-73.

25. Turner CG, Klein JD, Wang J, et al. The amniotic fluid as a source of neural stem cells in the setting of experimental neural tube defects. Stem Cells Dev 2013;22:548-53.

26. Li H, Gao F, Ma L, et al. Therapeutic potential of in utero mesenchymal stem cell (MSCs) transplantation in rat foetuses with spina bifida aperta. J Cell Mol 
Med 2012;16:1606-17.

27. Sharma AK, Bury MI, Fuller NJ, et al. Cotransplantation with specific populations of spina bifida bone marrow stem/progenitor cells enhances urinary bladder regeneration. Proc Natl Acad Sci U S A 2013;110:4003-8.
28. Okano H, Nakamura M, Yoshida K, et al. Steps toward safe cell therapy using induced pluripotent stem cells. Circ Res 2013;112:523-33.

29. Okano H, Yamanaka S. iPS cell technologies: significance and applications to CNS regeneration and disease. Mol Brain 2014;7:22. 